\section{Roche}

\section{Metastasiertes kolorektales Karzinom}

\section{Bevacizumab steigert R0-Resek- tionsrate und führt zu deutlicher Überlebensverlängerung}

Bevacizumab (Avastin ${ }^{\circledR}$ ), kombiniert mit einer Chemotherapie, steigert im Vergleich zu Plazebo die R0-Resektionsrate. Wie auf dem diesjährigen amerikanischen Krebskongress (ASCO) vorgestellte Daten zeigen, ist das 2-Jahres-Überleben der R0-resezierten und dann mit Bevacizumab weiterbehandelten Patienten höchst beeindruckend.

Bei Patienten mit kolorektalem Karzinom ist die Leber das am häufigsten von Metastasen befallene Organ. In 15\% der Fälle liegen schon zum Zeitpunkt der Diagnose Lebermetastasen vor, und bei weiteren 40-50\% der Patienten treten sie im Verlauf der Erkrankung auf. Die einzige Möglichkeit, diese Patienten zu heilen, besteht in der vollständigen operativen Entfernung (R0-Resektion) der hepatischen Metastasen. Bei Patienten mit zunächst nicht resektablen Metastasen kann durch eine systemische Therapie Resektabilität erzielt werden, wenn die Patienten auf die Therapie ansprechen.

\section{Steigerung des 2-Jahres-Überlebens im} RO-resezierten Kollektiv

In der Phase-III-Studie NO16966 erhielten 1401 Patienten mit lokal fortgeschrittenem oder metastasiertem kolorektalem Karzinom die Chemotherapie FOLFOX oder XELOX und randomisiert zusätzlich Bevacizumab oder Plazebo. Das Erzielen von Resektabilität war dabei kein erklärtes Studienziel - eine geplante größere Operation stellte vielmehr ein Ausschlusskriterium dar. Dennoch konnten 59 Patienten im Bevacizumab-Arm und 43 Patienten im Plazebo-Arm mit kurativer Absicht operiert werden. Wie die postoperative, histologische Auswertung zeigte, betrug die R0-Resektionsrate im Bevacizumab-Arm 6,3\% (44 von 699 Patienten (Intent-to-treatPopulation)) versus 4,9\% (34 von 701 Patienten) im Plazebo-Arm. Bei den Patienten, bei denen die Leber das einzige von Metastasen befallene Organ war, lag die R0-Resektionsrate bei 12,3\% (26 von 211 Patienten) im Bevacizumab-Arm versus $11,6 \%$ (24 von 207 Patienten) im Vergleichsarm. Das 2Jahres-Überleben betrug in diesem Fall im Bevacizumab-Arm 92,3\% versus 79,0\% im Plazebo-Arm. Zum Vergleich: Konnte keine R0-Resektion durchgeführt werden, betrug das 2-Jahres-Überleben der Patienten, die nur Lebermetastasen aufwiesen, $45,1 \%$ im Bevacizumab-Arm gegenüber $41,4 \%$ im Plazebo-Arm [1].

\section{Beobachtungsstudie First BEAT bestätigt RO-Resektionsrate und lange Überlebenszeit}

In der Beobachtungsstudie First BEAT, die die Sicherheit und Wirksamkeit von Bevacizumab in Europa und anderen Ländern (außer USA) untersuchte, wurden chirurgische Interventionen und ihr Ergebnis prospektiv erfasst. An der Studie nahmen insgesamt 1914 Patienten teil. Jeder Arzt konnte selbst entscheiden, mit welchem Chemotherapie-Regime er Bevacizumab kombinierte. 12\% (225 Patienten) der Patienten wurden in kurativer Absicht operiert. Empfohlen wurde ein Zeitraum von 6 bis 8 Wochen zwischen der letzten Bevacizumab-Gabe und der Operation. Die R0-Resektionsrate betrug insgesamt 9,0\% (173 Patienten). War die Leber das einzige von Metastasen befallene Organ, konnten 15,2\% der Patienten (107 Patienten) mit kurativer Absicht operiert werden. Die R0-Resektionsrate lag in diesem Fall bei $12,1 \%$ (85 Patienten). Ernste Wundheilungsstörungen wurden nur bei 1,3\% der operierten Patienten beobachtet, ernste Blutungen nur bei $0,4 \%$. Das 2-Jahres-Überleben der Patienten, bei denen die Lebermetastasen R0-reseziert werden konnten, betrug $89 \%$ - gegenüber $47 \%$, wenn nicht in kurativer Absicht operiert wurde.

\section{Literatur}

1 Cassidy et al. ASCO 2008; Abstract 4022

Weitere Informationen bei medical relations $\mathrm{GmbH}$

Moritz Keller

Hans-Böckler-Str. 46, 40764 Langenfeld Tel. +49 2173 9769-28, Fax -49 moritz.keller@medical-relations.de

\title{
Ticker+++ Ticker+++ Ticker+++ Ticker+++ Ticker+++ Ticker+++ Ticker+++
}

Bayer HealthCare AG. Die staatliche Arzneimittelzulassungsbehörde in China hat Nexavar ${ }^{\circledR}$ (Wirkstoff: Sorafenib) für die Behandlung von inoperablen oder metastasierenden Formen von Leberkrebs zugelassen. Nexavar ist das erste Arzneimittel, das die allgemeine Überlebensdauer bei Patienten mit dieser Erkrankung signifikant verlängern kann und nun als erste zugelassene systemische Therapie für diese Erkrankung in China eingesetzt werden darf

Weitere Informationen be

Bayer Schering Pharma AG

Dr. Jost Reinhard

jost.reinhard@bayerhealthcare.com
Wyeth Pharma GmbH. Aufgrund der bedeutenden Fortschritte der vergangenen Jahre in der Therapie des Nierenzellkarzinoms hat Wyeth Pharma eine neue Patientenbroschüre veröffentlicht, die aktuelle Informationen zur Erkrankung und zu den neuen zielgerichteten Therapieoptionen enthält. Ärzte haben ab sofort die Möglichkeit, die Broschüre per Post anzufordern oder im Internet auf www.torisel.de herunterzuladen.

Weitere Informationen bei

Wyeth Pharma GmbH

Mirja Kaupmann

KaupmaM@wyeth.com
Novartis Pharma GmbH. Die European Society for Medical Oncology (ESMO) hat ihre klinischen Empfehlungen zur Behandlung von gastrointestinalen Stromatumoren (GIST) aktualisiert. Die Gabe von 400 mg Imatinib täglich bleibt weiterhin die empfohlene Erstlinientherapie für GIST-Patienten. Des Weiteren wird nun erstmals die Durchführung einer Mutationsanalyse gefordert, deren Ergebnis insbesondere für Patienten mit Exon 9 Mutationen therapeutische Konsequenzen hat

Weitere Informationen be

Novartis Pharma GmbH

Dr. Irene Roth

irene.roth@novartis.com 